\title{
Cellular Features of the Fronds and Turions in Spirodela polyrhiza
}

InSun Kim*

\author{
Department of Biology, College of Natural Sciences, Keimyung University, Daegu 704-701, Korea
}

\author{
*Correspondence to: \\ Kim IS, \\ Tel: $+82-53-580-5305$ \\ Fax: +82-53-580-5305 \\ E-mail: botany@kmu.ac.kr
}

Received October 31, 2013

Revised December 9, 2013

Accepted December 9, 2013

\begin{abstract}
Structural aspects of highly reduced vegetative organs in the aquatic Spirodela polyrhiza were examined using scanning and transmission electron microscopy. The study focused mainly on young and mature fronds with turions and their cellular features were compared. Mature fronds were composed of thin-walled chlorenchyma with highly vacuolated cells; most of which were frequently occupied by either tanniferous deposits or various crystals. Fronds of photoautotrophic offspring were produced from the meristematic region of the reproductive pockets within mother fronds, where they remained until separation. Moderate degrees of wall ingrowth and plasmalemma proliferation were detected briefly in the epidermis of daughter fronds during early development. Vascular tissues were generally much reduced, but air chambers were well-established in fronds. Chloroplasts having grana with several thylakoids were distributed throughout the plant, but starch grains were encountered frequently in the mesophyll chloroplasts of younger fronds and initial stage of the turion. Their cytoplasm was dense with small vacuoles in most cases. Further, big starch grains, up to several microns, occupying most of the plastid volume were formed in the turion prior to sink for overwintering. Plasmodesmata were numerous in the examined tissues, except mature turions, suggesting a symplastic pathway of the metabolites within body.
\end{abstract}

Key Words: Spirodela polyrhiza, Ultrastructure, Frond, Turion, Electron microscopy

\section{INTRODUCTION}

Spirodela polyrhiza, a giant duckweed, of the family Lemnaceae is a small, free-floating aquatic species and well-known for a peculiar mode of vegetative reproduction. Fronds are the leaflike structures derived from fused stems and leaves (Landolt, 1986, 1998; Lemon \& Posluszny, 2000), with a multiple root system on each frond (Kim, 2007). A mother frond produces daughter fronds from two meristematic regions several times during growth, but turions are also formed within mother frond toward the end of growing season as dormant overwintering structures. The frond and/or turion primordia develop from those meristematic tissues and the repetition of primordial growth within mother fronds leads to characteristic vegetative reproduction in this species.

The frond of S. polyrhiza has drawn attention among aquatic species, since several generations of daughter fronds originated by peculiar mode from mother fronds. With light or electron microscopy, the nature of pigmentation (Formin et al., 1992), morphological patterns (Landolt, 1998), frond and turion anatomy (Appenroth \& Bergfeld, 1993; Kim \& Kim, 2000; Kwak \& Kim, 2008), and frond abscission were studied in several Spirodela species. Structural features of another genus in the same family, Lemna (Melaragno \& Walsh, 1976; Walsh \& Melaragno, 1976; Echlin et al., 1982; Echlin, 1992), have drawn some attention, but rather their root system was studied extensively. Formin et al. (1992) also revealed the grana features in chloroplasts while surveying the greening process of etiolated giant duckweed. Landolt (1998) and Lemon and Posluszny (2000) reported detailed structural information on the Spirodela species at low magnification while describing relationships among taxa in the Lemnaceae. Numerous studies have been carried out using plants of $S$. polyrhiza in various fields, but structural features mainly

(a) This is an open-access article distributed under the terms of the Creative Commons Attribution Non-Commercial License (http://creativecommons.org/licenses/by-nc/3.0) which permits unrestricted noncommercial use, distribution, and reproduction in any medium, provided the original work is properly cited.

Copyrights @ 2013 by Korean Society of Microscopy 
focused on organelles at the cellular level have been rather scarce in this species. Hence, the present study has been performed a comparative analysis of the ultrastructure of the vegetative $S$. polyrhiza. As in S. polyrhiza, some hydrophytes form turions (Weber \& Noodén, 2005) for overwintering during cold season. The turions, dormant structures adapted for survival during the winter by sinking to the bottom of the water, were also included for comparison. Structural aspect of the root system was excluded in the study, since it was discussed in detail as a separate paper (Kim, 2007).

\section{MATERIALS AND METHODS}

Approximately 20 to 30 mature plants of S. polyrhiza (L.) Schleiden were collected from the pond located at Keimyung University. Mother fronds having daughter fronds during the month of July, and mature fronds with turions in late September were sampled and processed by the following procedures.

Mother fronds having two to three daughter fronds and mature fronds with turions were fixed for 2 to 4 hours in $3 \%$ glutaraldehyde at room temperature and washed three times in $0.1 \mathrm{M}$ sodium phosphate buffer ( $\mathrm{pH}$ 7.2). Fixed tissue samples were dehydrated in a graded series of acetone followed by the critical point drying with liquid carbon dioxide (Emitech K850; Emitech Ltd., Ashford, UK). The processed samples were mounted directly on aluminum stubs using double sided adhesive tape and coated about 10 $\mathrm{nm}$ with gold/palladium in a sputter coater (Emitech K550X; Emitech Ltd.). The coated samples were examined with Hitachi S-4200 scanning electron microscope in Korea Basic Science Institute (KBSI), Daegu Center in Korea.

For transmission electron microscopy (TEM), mesophyll tissues of mother fronds having daughter fronds and mature fronds with turions were sliced under the dissecting microscope and immediately subjected to the following procedures: approximately 4 to $6 \mathrm{~mm}^{2}$ sections of tissue from each species were fixed in $3 \%$ glutaraldehyde in $0.1 \mathrm{M}$ phosphate buffer ( $\mathrm{pH} 7.2$ ) at room temperature for 3 hours (Kim \& Kim, 2000). The fixed tissues were rinsed three times in the same buffer and post-fixed in 2\% osmium tetroxide at $4^{\circ} \mathrm{C}$ overnight. After three rinses in the buffer, they were dehydrated through a graded acetone series, substituted, and embedded in low-viscosity Spurr resin. About 60 to $90 \mathrm{~nm}$ ultrathin sections were cut with the Ultracut-S ultramicrotome (Leica, Deerfield, IL, USA) using a diamond knife. Grids coated with $0.3 \%$ chloroform-diethanol formvar were used for the sections. They were double stained with $2 \%$ uranyl acetate and $0.5 \%$ lead citrate for 30 to 45 minutes each and examined with the Hitachi-H 7100 TEM in the KBSI, Daegu Center.

\section{RESULTS AND DISCUSSION}

Plants of S. polyrhiza are considered to be poorly differentiated as they produce only small fronds, connective tissues, and roots when matured. As the major photosynthetic organ, fronds produced offspring in the reproductive pocket. The meristematic regions examined in the reproductive pockets of mother fronds produced offspring fronds vegetatively (Fig. 1A) throughout the growing season. The offspring fronds remained within the pocket while being protected by a layer of foliage sheath until separation (Fig. 1B). The sheath was a 1-celled layer having a dense cytoplasm with chloroplasts and numerous mitochondria without intercellular spaces (Fig. 1C). All of the mesophyll cells in the daughter fronds formed while they were still in the pockets. The daughter fronds had limited air spaces and consisted of four to five layers of mesophyll containing many chloroplasts loaded with starch grains (Fig. 1D). The offspring became photoautotrophic fairly early while they still remained in the mother frond. The epidermal cells, as well as the internal tissues of the entire plant, contained numerous chloroplasts, indicating the occurrence of photosynthesis throughout the plant body. Individual grana stacks in chloroplasts exhibited three to several thylakoids. Granal chloroplasts have been reported in species of Lemna (Beaumont et al., 1980), Spirodela (Formin et al., 1992; Kim \& Kim, 2000; Kim, 2007; Kwak \& Kim, 2008), and Wolffia (Bernard et al., 1990; White \& Wise, 1998) within the family. However, unlike the chloroplasts which lack starches in the meristematic cells of Wolffia, the immature fronds and developing turions in S. polyrhiza had chloroplasts heavily loaded with starches. The accumulation of starch grains in young frond cells suggested that they might have an important role in carbohydrate storage, triggering rapid growth. In contrast, such accumulation was not found in mature, old fronds. The fact that mesophyll chloroplasts in mature fronds usually did not accumulate starches implies the possible transfer of photosynthates from the mesophyll to other parts of the plant soon after production. Numerous plasmodesmata developed between cells within the reduced body further supported the symplastic transport of nutrients from photosynthetic cells to developing tissues.

The epidermal cells were much like the underlying chlorenchymatous cells in ultrastructure, showing numerous chloroplasts with 3 to 6 thylakoids/granum, small lipid droplets, and starch grains (data not shown). Single or grouped plasmodesmata commonly occurred between epidermal cells or between epidermal and mesophyll cells. While chloroplasts with starch grains were prominent in the meristematic daughter frond, proplastids were also occasionally seen at this stage. Large starch grains remained for some time in expanding mesophyll cells. Thus, granal chloroplasts with starch grains were already present in 
daughter fronds that had never been exposed to sunlight. A moderate degree of wall ingrowth and plasmalemma proliferation (Fig. 1E and F) was encountered in the abaxial epidermis of daughter frond that was exposed to sunlight, but this feature was retained only for a short period of time during early development. After the release, mesophyll cells of the daughter frond underwent rapid vacuolization, while the chloroplasts retained large starch grains for some period of time. The abaxial epidermis of the giant duckweed differed considerably from the cutinized adaxial epidermis in transverse sections.

Other morphological features readily noted during frond differentiation were the development of aerenchyma and the formation of tannin cells. As the mesophyll cells enlarged, intercellular spaces and air chambers became prominent in the abaxial surface of maturing fronds. Air chambers of 7 to $18,156 \times 185 \mu \mathrm{m}$ (length $\times$ width) in average size, transversely bisected the fronds at somewhat irregular intervals along the hypodermis in the abaxial epidermis, where cells were much smaller than in the adaxial epidermis. Two adjacent air chambers were separated by a one cell-thick parenchymapartitioned wall.

The limited intercellular spaces within daughter fronds soon expanded and formed about 13 lacuna with a width range of 113 to $205 \mu \mathrm{m}$ and an average length of $156 \mu \mathrm{m}$ in mature fronds. Since the diameter of air chambers commonly fell within the range of 100 to $300 \mu \mathrm{m}$, the lacuna formed in mother fronds were clearly air chambers. Aerenchyma in Spirodela is thought to provide buoyancy and enables the diffusion of gases to cells (Klich et al., 1985); more specifically
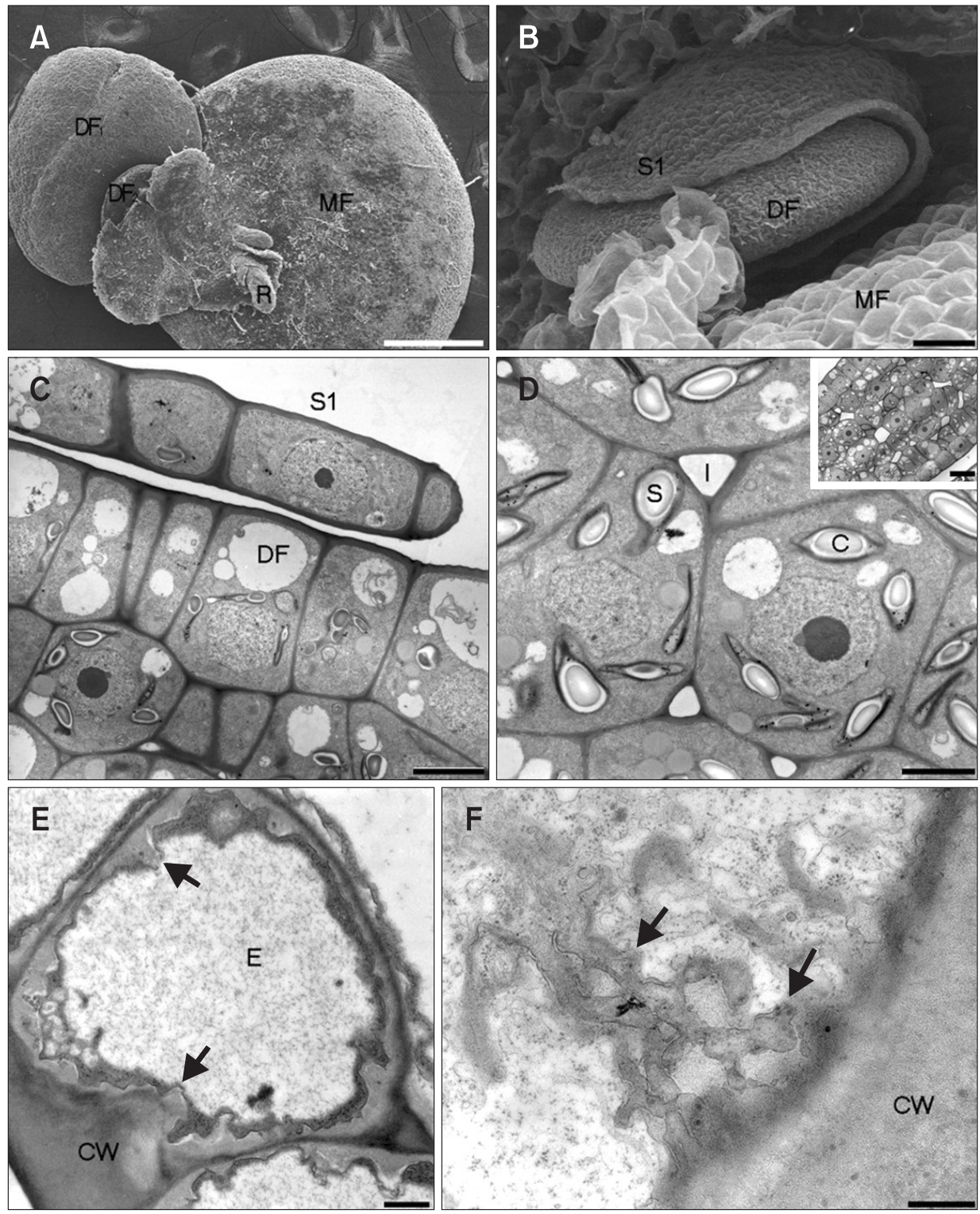

Fig. 1. Structural features of daughter fronds. (A) Mother frond releasing two offspring fronds $\left(\mathrm{DF}_{1}, \mathrm{DF}_{2}\right)$. Scale bar $=500$ $\mathrm{nm}$. (B) A reproductive pocket containing a developing daughter frond protected by a foliage sheath. Scale bar $=20 \mu \mathrm{m}$. (C) Cross-section partially showing sheath and daughter frond. Scale bar=3 $\mu \mathrm{m}$. (D) Mesophyll cells containing numerous chloroplasts loaded with starch grains. Scale bar $=3 \mu \mathrm{m}$. Inset: paradermal section of a daughter frond as in Fig. 1B. Scale bar $=10 \mu \mathrm{m}$. (E) Moderate degree of wall ingrowth (arrows, scale bar $=300$ $\mathrm{nm}$ ) and (F) plasmalemma proliferation (arrows, scale bar $=500 \mathrm{~nm}$ ) in the abaxial epidermis. DF, daughter frond; R, root; MF, mother frond; S1, sheath; S, starch grain; I, intercellular space; C, chloroplast; E, epidermis; CW, cell wall. 
functioning as a gas exchange chamber containing both $\mathrm{O}_{2}$ and $\mathrm{CO}_{2}$, which facilitates photosynthesis (Kuo et al., 1990). Mesophyll cells were large with thin walls, each containing thin cytoplasm with huge vacuole occupying most of the lumen (Fig. 2A). Chloroplasts usually contained 5 to 9 thylakoids/granum, plastoglobuli, and starch grains (Fig. 2B). Vacuoles of mesophyll cells were frequently filled either with prominent deposits of tannins (Fig. 2C and D), druse crystals, presumably calcium oxalate, or bundles of short and long raphides (Fig. 2E and F). Large amounts of anthocyanin pigments were also found in certain cells of the abaxial epidermis.

Frequently unbranched simple plasmodesmata, sometimes as a group, occurred between adjacent cells (Fig. 3A). Welldeveloped plasmodesmata were distinguished in the thin primary pit fields of mesophyll cell located close to the connective tissue, where thick electron-dense walls abutted intercellular spaces (Figs. 3A and B). The plamodesmata were well-established throughout the expanding frond (Fig. 3C). However, complete plasmodesmatal connections between the epidermis and mesophyll cells disintegrated and no cell wall ingrowth was detected in epidermal cells at maturity. When
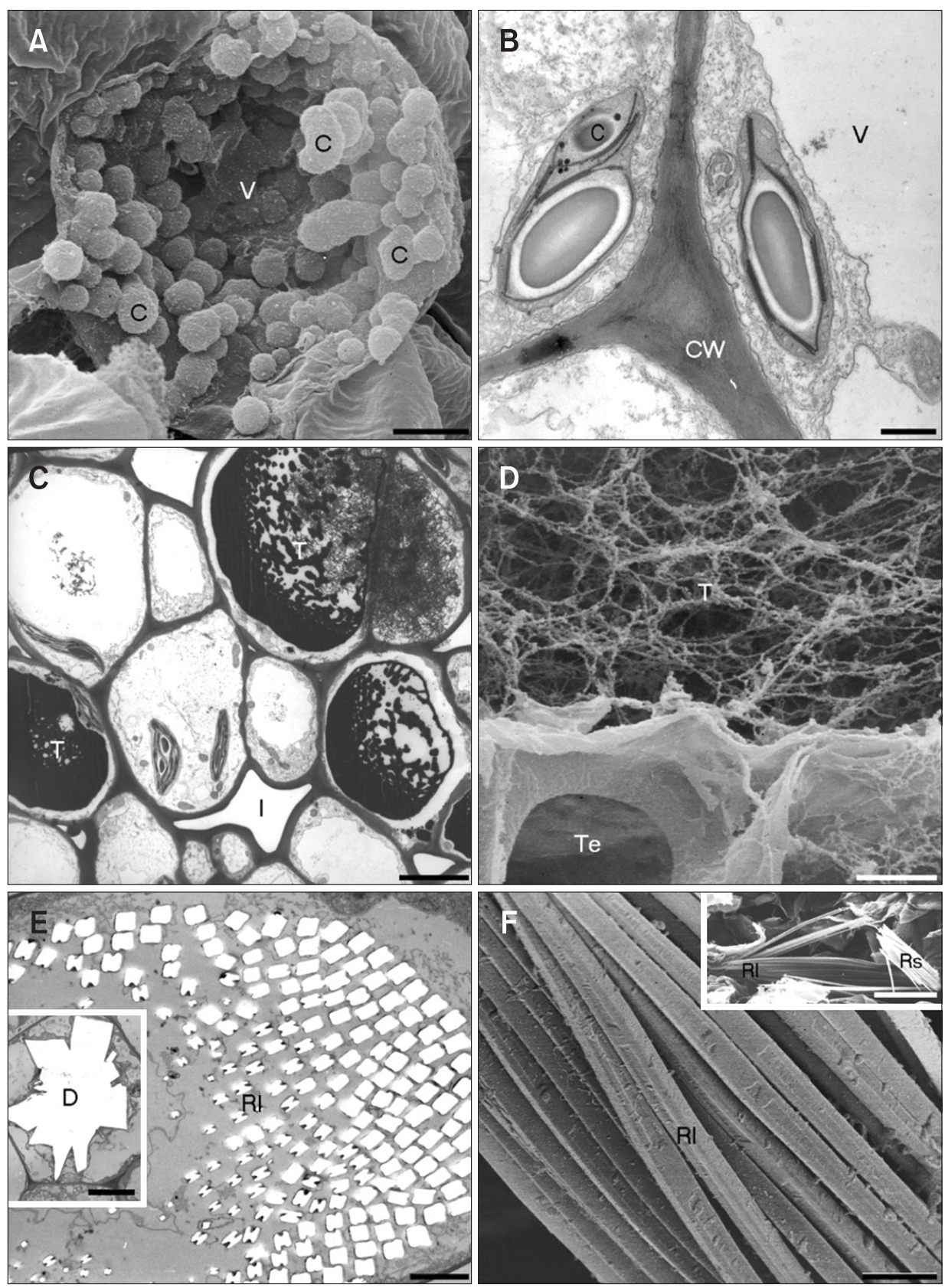

Fig. 2. Mesophyll cells of mature fronds. (A) Mesophyll cell showing a large vacuole with peripherally arranged organelles. Scale bar $=1.5 \mu \mathrm{m}$. (B) Peripherally arranged chloroplasts. Scale bar $=600 \mathrm{~nm}$. (C) Idioblastic tannin cells. Scale bar $=2 \mu \mathrm{m}$. (D) Portion of a tannin-accumulating cell adjacent to a tracheary element. Scale bar $=1 \mu \mathrm{m}$. (E) Transverse section of a raphide bundle within vacuole. Scale bar $=2$ $\mu \mathrm{m}$. Inset: druse crystal in cross section. Scale bar $=5 \mu \mathrm{m}$. (F) Close-up of long raphide crystals. Scale bar $=1.5 \mu \mathrm{m}$. Inset: bundles of short and long raphides. Scale bar=20 $\mu \mathrm{m}$. C, chloroplast; V, vacuole; CW, cell wall; T, tannin; I, intercellular space; Te, tracheary element; D, druse crystal; Rl, long raphide; Rs, short raphide. 
fronds were fully expanded, chloroplasts usually showed little or no starch grains, except for those in guard cells (Fig. 3D). There were three to five veins, mostly five in fronds, but the vascular system was fairly simple in structure. The veins were in direct contact with the mesophyll cells without any sheath cells (Fig. 3E). In general, one tracheary element developed along with $3(-4)$ sieve elements having P-type plastids, vesicles, and fibrils (Fig. 3F).

S. polyrhiza has been investigated in great deal especially physiological, biochemical and ecological areas, but a little is known concerning the structural features at the cell level during development. Thus, the present study attempted to reveal the structural characteristics of the frond and turion such as tissue differentiation and ultrastructure, employing electron microscopy. Moderate degree of mesophyll tissue differentiation was found in the frond, while the turion did not exhibit such differentiation. Within the tissue, about $37 \%$ to $45 \%$ of the cellular volume is occupied by the huge aerenchyma, but only about $9 \%$ to $15 \%$ is taken by the aerenchyma in the turion. The turion cells, especially turion primordium, derived from the frond cells and contained cytoplasm. At primordial stage, the cytoplasm was filled with plastids, mitochondria, endoplasmic reticulum, Golgi bodies, and microtubules. Plasmodesmata were also well developed among these cells, but they were not found in mature, old turions preparing dormancy. The new fronds arose from such turions and began to grow rapidly for a new life cycle in spring. The turions are modified fronds in terms of ontogeny, although their tissues are thicker and smaller in size relative to the fronds. All these anatomical and ultrastructural features revealed in the study have been considered to be efficient for the structural organization and growth within S. polyrhiza plant despite of highly reduced body size.

\section{CONCLUSIONS}

Structural aspects of young and mature fronds with turions were studied and their cellular features were compared. All
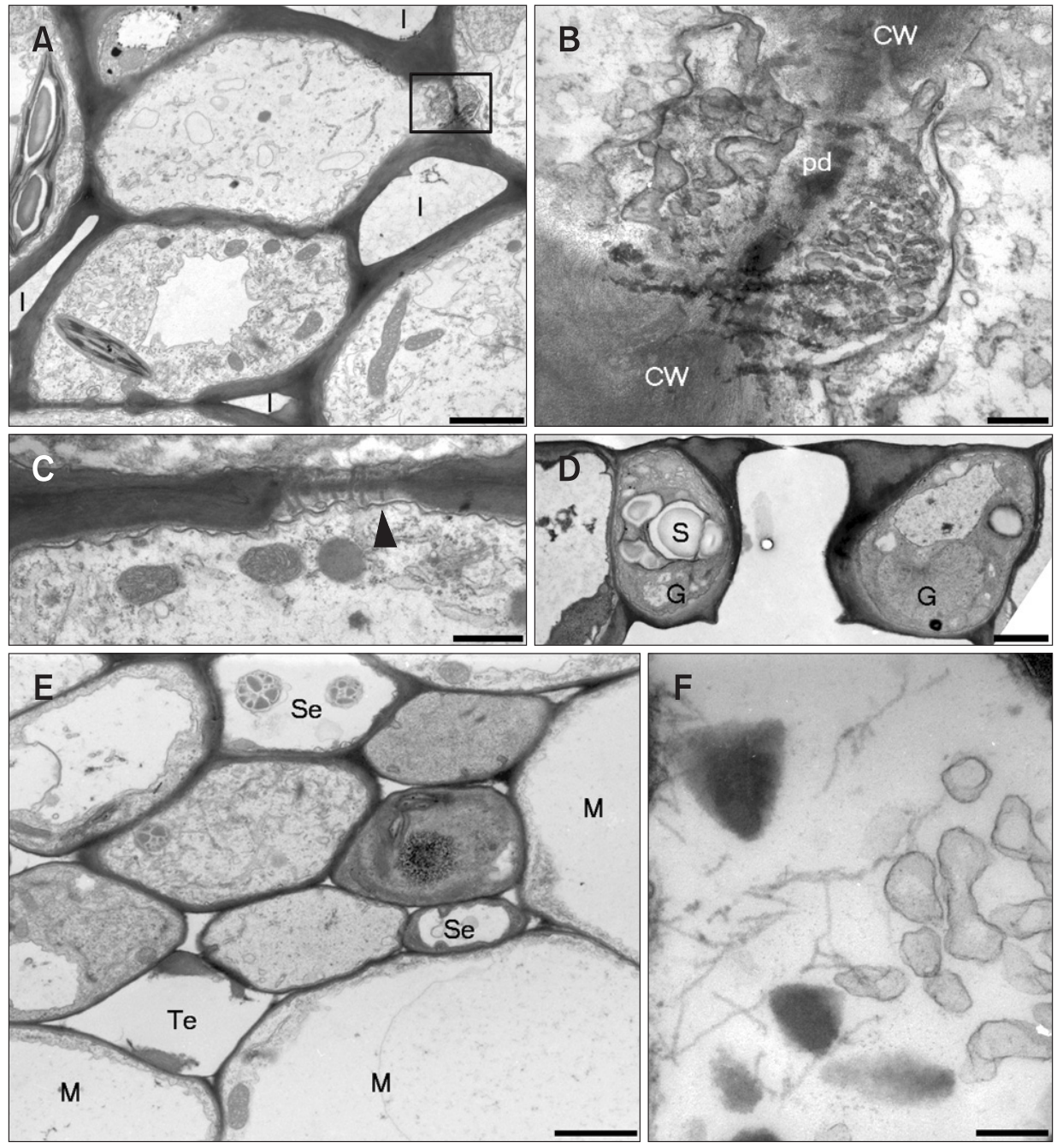

Fig. 3. Structural features of mature fronds. (A) Electron-dense thick cell walls abutting intercellular spaces near the connective tissue. Scale bar $=2 \mu \mathrm{m}$. (B) Close-up of a region (box) in Fig. 3A showing wellestablished plasmodesmata between cells. Note the thick cell wall. Scale bar $=250 \mathrm{~nm}$. (C) Primary pit field with plasmodesmata (arrowhead). Scale bar=600 nm. (D) Chloroplasts having starch grains in guard cells. Scale bar $=2 \mu \mathrm{m}$. (E) A vein showing one tracheary element and a few sieve elements. Note the absence of bundle sheath around the vein. Scale bar $=2 \mu \mathrm{m}$. (F) Enlarged view of a sieve plastid. Scale bar=200 nm. I, intercellular space; CW, cell wall; pd, plasmodesmata; S, starch grain; G, guard cell; Se, sieve element; $\mathrm{Te}$, tracheary element; M, mesophyll cell. 
fronds were photosynthetic regardless of developmental stages including unexposed offspring fronds and turions. Mesophyll tissues of the mother fronds were thin-walled chlorenchyma with highly vacuolated cells, while those of the offsprings and turions were filled with dense cytoplasm at early growth. Frequently either tanniferous deposits or various crystals occupied the mesophyll cells. The photoautotrophic offspring derived from the meristematic region of the reproductive pockets within mother fronds, where they remained until separation. Moderate degrees of wall ingrowth and plasmalemma proliferation were detected briefly in the epidermis of daughter fronds during early development.
Vascular tissues were generally much reduced, but air chambers were well-established in fronds. Chloroplasts having grana with several thylakoids were distributed throughout the plant, but starch grains were encountered frequently in the mesophyll chloroplasts of younger fronds and initial stage of the turion. Their cytoplasm was dense with small vacuoles in most cases. Further, big starch grains, up to several microns, occupying most of the plastid volume were formed in the turion mesophyll prior to sink for overwintering. Plasmodesmata were numerous in the examined tissues, except mature turions, suggesting a symplastic pathway of the metabolites within the body.

\section{REFERENCES}

Appenroth K J and Bergfeld R (1993) Photophysiology of turion germination in Spirodela polyrhiza (L.) Schleiden XI. Structural changes during red light induced responses. J. Plant Physiol. 141, 583-588.

Beaumont G, Lord A, and Grenier G (1980) Effects physiologiques de l'atrazine doses subl tales sur Lemna minor. V. Influence sur l'ultrastructure des chloroplastes. Can. J. Bot. 58, 1571-1577.

Bernard F A, Bernard J M, and Denny P (1990) Flower structure, anatomy and life history of Wolffia australiana (Benth.) den Hartog \& van der Plas. Bull. Torrey Bot. Club. 117, 18-26.

Echlin P (1992) Low-Temperature Microscopy and Analysis, pp. 349-411, (Plenum Press, New York).

Echlin P, Lai C E, and Hayes T L (1982) Low-temperature X-ray microanalysis of the differentiating vascular tissue in root tips of Lemna minor L. J. Microsc. 126, 285-306.

Formin A V, Ladygin G, and Semenova G A (1992) Accumulation of pigments and formation of chloroplast ultrastructure in the process of greening of etiolated duckweed plants. Sov. Plant Physiol. 38, 483488.

Kim I (2007) Development of the root system in Spirodela polyrhiza (L.) Schleiden (Lemnaceae). J. PI. Biol. 50, 540-547.

Kim K and Kim I (2000) Structural aspect of the reduced free-floating hydrophyte, Spirodela polyrhiza. Korean J. Electron Microsc. 30, 233240.

Klich M G, Mújica M B, and Fernández O A (1985) The effect of gibberellic acid on the buoyancy of Spirodela intermedia W. Koch. Aquat. Bot.
21, 63-69.

Kuo J, Ridge W R, and Lewis S V (1990) The leaf internal morphology and ultrastructure of Zostera muelleri Irmisch ex Aschers (Zosteraceae). A comparative study of the intertidal and subtidal forms. Aquat. Bot. 36, 217-236.

Kwak M and Kim I (2008) Turion as dormant structure in Spirodela polyrhiza. Korean J. Microsc. 38, 307-314.

Landolt E (1986) The Family of 'Lemnaceae': A Monographic Study (Geobotanisches Institut der ETH, Zürich).

Landolt E (1998) Anatomy of the Lemnaceae (duckweeds). In: Extreme Adaptations in Angiospermous Hydrophytes, eds. Landolt E, JagerZurn I, and Schnell RAA, pp. 1-127, (Borntraeger, Berlin).

Lemon G D and Posluszny U (2000) Comparative shoot development and evolution in the Lemnaceae. Int'l. J. Plant Sci. 161, 733-748.

Melaragno J E and Walsh M A (1976) Ultrastructural features of developing sieve elements in Lemna minor L. the protoplast. Amer. J. Bot. 63, 1145-1157.

Walsh M A and Melaragno J E (1976) Ultrastructural features of developing sieve elements in Lemna minor L. sieve plate and sieve areas. Amer. J. Bot. 63, 1174-1183.

Weber J A and Noodén L D (2005) The cause of sinking and floating in turion of Myriophyllum verticillatum. Aquat. Bot. 83, 219-226.

White S L and Wise R R (1998) Anatomy and ultrastructure of Wolffia columbiana and Wolffia borealis, two nonvascular aquatic angiosperms. Int'l. J. Plant Sci. 159, 297-304. 